\title{
Phonon squeezing via correlations in the superconducting electron-phonon interaction
}

\author{
T. Hakioḡu, V.A. Ivanov, ${ }^{*}$ A.S. Shumovsky, and B. Tanatar \\ Department of Physics, Bilkent University, TR-06533 Bilkent, Ankara, Turkey
}

(Received 14 September 1994)

\begin{abstract}
Superconductivity in the conventional BCS model with correlated squeezed phonons is discussed. It is shown that the energy gap and the critical temperature are maximally enhanced in an optimum and finite range of squeezed coupling. For finite-squeezed coupling the ratio $2 \Delta / T_{c}$ becomes coupling-constant dependent and increases beyond the BCS value of 3.53. Ion-mass dependence of the squeezed coupling constant can yield variations of the isotope exponent from its conventional BCS value of 0.5 .
\end{abstract}

\section{INTRODUCTION}

The great success of the BCS model in the conventional theory of superconductivity has been advanced by the discovery of the mechanism of attractive electronelectron interaction mediated by the phonon exchange. ${ }^{1}$ Early experimental investigations of isotope effect ${ }^{2,3}$ demonstrated the importance of the lattice vibrations which then resulted in the theoretical formulation by Fröhlich ${ }^{4}$ and Bardeen. ${ }^{5}$ Following this, the universality of $2 \Delta / T_{c}$ and other important results have been understood. Today direct phonon exchange is known to be insufficient for the understanding of a number of conventional superconductors as well as of high- $T_{c}$ materials although the phonons are undoubtedly known to participate in the formation of the superconducting state. ${ }^{6}$ First of all a considerable change in the isotope effect and the other phonon-related properties should be emphasized. ${ }^{7,8}$

In the investigations of high- $T_{c}$ superconductivity a tremendous number of possible pairing mechanisms are discussed to explain especially the anomalies in the isotope data. For instance in charged Bose liquid superconductivity $^{9}$ the isotope exponent $\alpha$ is negative and quite small. The Hubbard model with $e-p h$ interactions of the Fröhlich type predicts positive $\alpha$ (Ref. 10) whereas in the overdoped case one finds $\alpha<0$ (Ref. 11) always contradicting the experimental data. The van Hove scenario ${ }^{12}$ also cannot explain the isotope data properly. It should be noticed from these remarks that there is no known universality between $\alpha$ and $T_{c}$.

In the conventional theory of superconductivity based on the Fröhlich model of the low-energy phonon exchange as well as in many models of high- $T_{c}$ superconductivity involving phonon degrees of freedom, ${ }^{6}$ the linear form of the interaction corresponding to the harmonic potential approximation is considered. At the same time, we know that the phonon correlations exist in some real superconducting materials because of strong anharmonicities and other reasons. ${ }^{13}$ Among those one can briefly mention the IR and Raman spectra, the Fano effect ${ }^{14}$ including both the phonon and the electron components, the photo-induced absorption, ${ }^{15}$ the photo-induced conductivity in the mid-IR range, ${ }^{16}$ and the photo-induced superconductivity. ${ }^{17}$ The 1-2-3 lattice deformation at $T_{c}$ (Ref. 18) and the recent discovery of the generation of coherent phonons upon photo-induced pair breaking ${ }^{19}$ are evidence of a new kind of phonon state in unconventional superconductors. Although there is no direct evidence that structural lattice instability and high critical temperature are correlated, there is some room in theories favoring electron-phonon interaction for possible indirect influence of unusual phonon dynamics on the phonon ground state, hence on the $T_{c}$. It is important to know what kind of changes in the superconducting properties can be modeled in the context of phonon correlations.

In the present paper we consider the simplest case when the electron pairing is generated by the Fröhlich interaction with correlated phonons. Our formulation differs from that of $\mathrm{Zheng}^{20}$ in the study of the isotope effect. In order to understand the isotope anomalies resulting from the phonon correlations the mass dependence of the squeezed coupling has to be explicitly taken into account. This requires the squeezed phonon coupling constant to be kept in the calculations rather than treating it as an internal variational parameter. The organization of the paper is as follows. In Sec. II we introduce the BCS Hamiltonian including a simple model for twophonon correlations and present its solution. In Sec. III the effect of these correlations on the isotope exponent is discussed. Finally we conclude with a brief summary of certain physical examples including recently discovered borocarbide and boronitride superconductors. The reliability of this simple model within the context of weakcoupling regime and its extension to strong coupling is also mentioned.

\section{BCS HAMILTONIAN WITH PHONON CORRELATIONS}

In strongly interacting superconducting electronphonon narrow-band systems it is already known that the squeezed vacuum phonon ground state lowers the superconducting ground-state free energy by reducing the localization effects resulting from the polaronic nar- 
rowing of the electron band. ${ }^{20,21}$ Bipolaronic squeezing was also applied to high-temperature superconductors. ${ }^{22}$ Zheng has shown ${ }^{20}$ that in the strongly correlated electron systems with strong electron-phonon interaction, a new phonon ground state (i.e., squeezed state) is energetically favored against the polaron ground state. This new ground state is known from the parametric processes in quantum optics ${ }^{23}$ and is characterized by a supergaussian distribution with enhanced fluctuations in the number of particles in comparison to the most familiar equilibrium Bose-Einstein distribution. It was later shown ${ }^{24}$ that a correlated squeezed vacuum state of phonons in the momentum space is energetically favored in the same model as opposed to the uncorrelated one as discussed in Ref. 20. Anharmonic lattice effects are known to create phonon correlations. If such cases are in question for a conventional superconductor the deviations from the conventional BCS properties, in particular from the isotope effect, can be parametrized in terms of such correlations. For this purpose we start with the Fröhlich Hamiltonian with the simplest anharmonic phonon interaction which effectively includes two-phonon correlations as

$$
\begin{aligned}
\mathcal{H}= & \sum_{p, \sigma} \xi_{p} c_{p, \sigma}^{\dagger} c_{p, \sigma}+\sum_{q} \omega_{q} b_{q}^{\dagger} b_{q} \\
& +\sum_{q, k, \sigma} g_{q} c_{k, \sigma}^{\dagger} c_{k+q, \sigma}\left(b_{-q}+b_{q}^{\dagger}\right) \\
& +\sum_{q}\left(\kappa_{q} b_{q} b_{-q}+\kappa_{q}^{*} b_{q}^{\dagger} b_{-q}^{\dagger}\right)
\end{aligned}
$$

where $c_{p, \sigma} .\left(c_{p, \sigma}^{\dagger}\right)$ and $b_{q}\left(b_{q}^{\dagger}\right)$ are the fermion and phonon annihilation (creation) operators, and $\xi_{p}$ and $\omega_{q}$ are the single-particle electron and phonon energies, respectively. Here $g_{q}$ describes the Fröhlich-type electron-phonon interaction and $\kappa_{q}=\left|\kappa_{q}\right| e^{i \theta_{q}}$ describes the degree of correlations between the phonon states. In connection with quantum optics we will use the term squeezed coupling for $\kappa_{q}$. Obviously mechanisms which would give rise to the process in (1) are forbidden on grounds of energy conservation if the phonon subsystem is in thermal equilibrium with the rest of the system. However (1) can effectively represent correlations between the creation (annihilation) of two acoustic modes ${ }^{25}$ in the mean field of an anharmonic ion potential. A more generalized version of (1) can describe the correlations between two different modes which can arise in the mixed case of strong lattice anisotropy and anharmonicity. ${ }^{26}$ Correlations described by the above Hamiltonian have the virtue that nonlinearity of the interaction between different phonon modes is effectively taken into account. The pure phonon part of this Hamiltonian can be diagonalized by the Bogoliubov transformation,

$$
b_{q}=\mu_{q} B_{q}+\nu_{q} B_{-q}^{\dagger} .
$$

Since $b_{q}, b_{q}^{\dagger}$ are Bose operators, the squeezed phonon operators $B_{q}, B_{q}^{\dagger}$ are such that $\left[B_{q}, B_{q}^{\dagger}\right]=1$ with $\left|\mu_{q}\right|^{2}-$ $\left|\nu_{q}\right|^{2}=1$. The coefficients are given by

$$
\mu_{q}=\frac{i}{\sqrt{2}}\left(\frac{\omega_{q}+\Omega_{q}}{\Omega_{q}}\right)^{1 / 2} e^{i \theta_{q} / 2}
$$

and

$$
\nu_{q}=\frac{i}{\sqrt{2}}\left(\frac{\omega_{q}-\Omega_{q}}{\Omega_{q}}\right)^{1 / 2} e^{i \theta_{q} / 2}
$$

with $\Omega_{q}=\sqrt{\omega_{q}^{2}-4\left|\kappa_{q}\right|^{2}}$. For most realistic cases softening of the phonon frequency occurs near the boundaries of the Brillouin zone. If the softening is caused by certain anharmonic modes then a nonzero squeezed coupling can be generated self-consistently. Then $\kappa_{q}$ is expected to have a non-negligible momentum dependence within the momentum region where a strong softening in the phonon spectrum is observed. ${ }^{27}$ On the other hand we can take $\theta_{q}=0$ without any loss of generality. After a little algebra and by using (1), (2), and (3), an effective Hamiltonian describing the correlated phonon exchange can be obtained as

$$
\begin{aligned}
\mathcal{H}= & \sum_{p, \sigma} \xi_{p} c_{p, \sigma}^{\dagger} c_{p, \sigma}+\sum_{q} \Omega_{q} B_{q}^{\dagger} B_{q} \\
& +\sum_{q, k, \sigma} g_{q}\left(\left|\mu_{q}\right|+\left|\nu_{q}\right|\right) c_{k, \sigma}^{\dagger} c_{k+q, \sigma}\left(B_{-q}+B_{q}^{\dagger}\right)
\end{aligned}
$$

Phonons can now be decoupled from the electrons by applying a second Bogoliubov transformation

$$
B_{q}=C_{q}+\gamma_{q} \rho_{-q}^{\dagger}
$$

where $\rho_{q}=\sum_{k, \sigma} c_{k, \sigma}^{\dagger} c_{k+q, \sigma}$ describes the momentumdependent electron-density operator. The transformed phonon operators are $C_{q}, C_{q}^{\dagger}$ which obey $\left[C_{q}, C_{q}^{\dagger}\right]=1$. Here we choose $\gamma_{q}$ as

$$
\gamma_{q}=-\frac{g_{q}}{\Omega_{q}}\left(\left|\mu_{q}\right|+\left|\nu_{q}\right|\right)
$$

in order to kill the linear term in $g_{q}$. Equations (5) and (6) constitute an analog of the Lang-Firsov unitary transformation for the weakly interacting electron-phonon system. Finally the decoupled Hamiltonian is

$$
\begin{aligned}
\mathcal{H}= & \sum_{p, \sigma} \tilde{\xi}_{p} c_{p, \sigma}^{\dagger} c_{p, \sigma}+\sum_{q} \Omega_{q} C_{q}^{\dagger} C_{q} \\
& +\sum_{q, k, \sigma} V_{q} c_{k, \sigma}^{\dagger} c_{k^{\prime}+q, \sigma^{\prime}}^{\dagger} c_{k+q, \sigma} c_{k^{\prime}, \sigma^{\prime}}
\end{aligned}
$$

In the calculation of the single-particle renormalized electron energy $\tilde{\xi}_{p}$ by correlated phonons there is a contribution from the Hartree term which originates from reorganizing the four electron interaction in the conventional normal ordered BCS form. Unlike the conventional BCS case, however, a part of this renormalization has an explicit momentum dependence through $\kappa_{q}$. Calculating this part of the renormalized single-particle electron energy in the logarithmic approximation using rectangular phonon density of states, we find 
$\tilde{\xi}_{p}=\xi_{p}-\Sigma_{p}, \quad$ with $\quad \Sigma_{p}=\lambda \kappa_{p} \ln \left|\frac{1+2 \kappa_{p} / \omega_{D}}{1-2 \kappa_{p} / \omega_{D}}\right|$

where $\omega_{D}$ is the Debye energy, $\lambda=\rho_{F}\left|g_{q}\right|^{2} / \omega_{D}$ is the dimensionless BCS coupling constant corresponding to $\kappa_{q}=0$, and $\rho_{F}$ is the electron density of states at the Fermi level. In the usual Fröhlich-BCS picture $\omega_{D}$ corresponds to the energy cutoff introduced for the attractive $e-p h$ interaction which together with $\lambda$ constitute the two physical parameters of the model. Their magnitudes are deduced from the $T_{c}$ and the spectral measurements. Therefore these parameters should not be confused with the unphysical (bare) ones defined in the Hamiltonian. Hence we neglect the renormalization of $\omega_{q}$ or $\Omega_{q}$ beyond Eqs. (3). ${ }^{28}$ by,

The effective electron-electron coupling is then given

$$
V_{q}=-\frac{\left|g_{q}\right|^{2}}{\Omega_{q}} \frac{\omega_{q}+\kappa_{q}}{\Omega_{q}}
$$

The Hamiltonian (7) is in the simple conventional BCS form with the renormalized single-particle electron energy and enchanced effective attractive electron-electron coupling. In Eq. (9) the enhancement factor is given by $\frac{\omega_{q}}{\Omega_{q}} \frac{\omega_{q}+\kappa_{q}}{\Omega_{q}}$.

The ground-state zero-temperature energy gap $\Delta$ and the corresponding transition temperature $T_{c}$ can be obtained using conventional methods. In order to obtain an analytic result we first replace $\kappa_{q}$ by its average $\kappa$ over the Fermi surface. However this approximation is justified only in the weak-coupling mean-field limit. ${ }^{27}$ The energy gap and the transition temperature are then given by

$$
\begin{aligned}
& 1=\lambda\left(\frac{1+\kappa / \omega_{D}}{1-4 \kappa^{2} / \omega_{D}^{2}}\right) \ln \left\{\frac{1-\Sigma_{\kappa} / \omega_{D}+\left[\left(1-\Sigma_{\kappa} / \omega_{D}\right)^{2}+\Delta^{2} / \omega_{D}^{2}\right]^{1 / 2}}{\Delta / \omega_{D}}\right\}, \\
& 1=\lambda\left(\frac{1+\kappa / \omega_{D}}{1-4 \kappa^{2} / \omega_{D}^{2}}\right)\left[\ln \frac{1-\Sigma_{\kappa} / \omega_{D}}{T_{c} / \omega_{D}} \tanh \frac{1-\Sigma_{\kappa} / \omega_{D}}{T_{c} / \omega_{D}}+\ln 1.13\right] .
\end{aligned}
$$

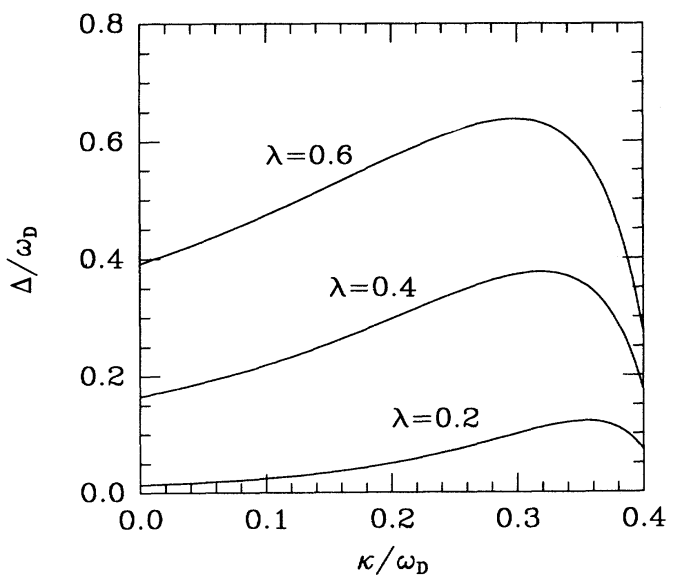

FIG. 1. Superconducting BCS energy gap as a function of squeezed coupling.

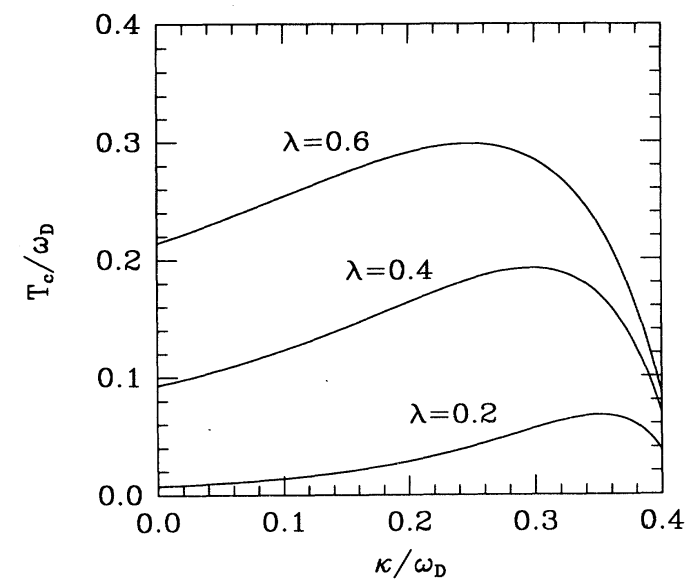

FIG. 2. Superconducting transition temperature as a function of squeezed coupling.

It is shown in Figs. 1 and 2 that for $\kappa \neq 0$ both the energy gap and the critical temperature monotonically increase until an optimum value $\kappa_{\text {opt }}$. For small coupling $\lambda \simeq 0.1$ the ratio $T_{c}\left(\kappa_{\text {opt }}\right) / T_{c}(0)$ can be as high as 100 . The same ratio becomes smaller very rapidly as $\lambda$ increases. For instance, for $\lambda=0.3$ that ratio is approximately 3.2 and for $\lambda=0.5$ it is near 1.6. As the squeezed coupling increases the electron band width implied by Eq. (8) is gradually narrowed. Around $2 \kappa / \omega_{D} \sim 1$ electrons become strongly localized due to the strong electron-phonon coupling, however in this limit these results are probably unreliable due to the weak-coupling approximation. Also in Fig. 3 we show the deviation of the ratio $2 \Delta / T_{c}$ from the standard BCS value 3.53 for increasing $\kappa$.

\section{THE ISOTOPE EFFECT}

Another interesting result of the existence of correlated phonons is the deviation of the isotope effect from its conventional behavior. The ion-mass dependence of $\kappa$

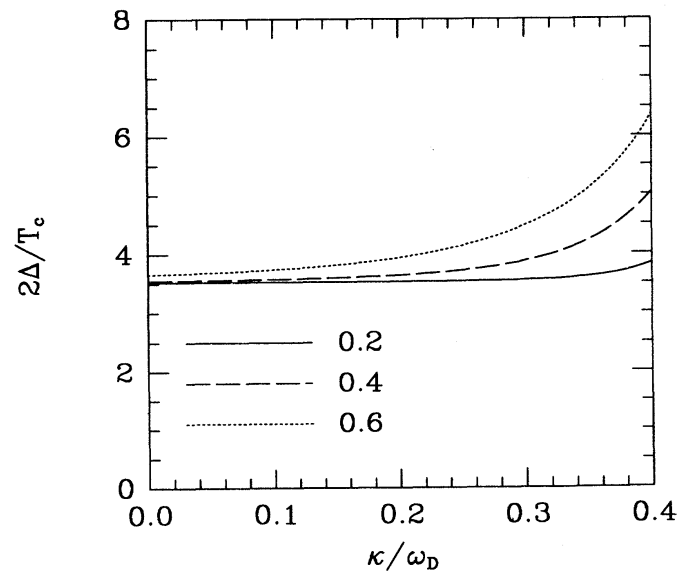

FIG. 3. The dimensionless BCS ratio $2 \Delta / T_{c}$ as a function of squeezed coupling. 
is subject to the nature of the mechanism which drives the phonon correlations. Here in order to examine the isotope effect we take up a particular case of a double-well anharmonic lattice potential. Using the self-consistent harmonic approximation ${ }^{29}$ (SCHA) it is possible to show that the mean-field, displaced-ionic potential implicit in Eq. (1) generates a dynamical squeezed coupling defined by $^{30}$

$$
\kappa_{q}=\frac{\left\langle V^{\prime \prime}\right\rangle}{2 M \omega_{q}}\left(\mu_{q}+\nu_{q}\right)^{2}
$$

where $\left\langle V^{\prime \prime}\right\rangle$ is the expectation value of the renormalized force constant evaluated in the phonon ground state of the double-well at zero temperature and $M$ is the ion mass. Since from Eq. (3) $\mu_{q}$ and $\nu_{q}$ depend on $\kappa_{q}$, Eq. (11) represents a self-consistency condition for $\kappa_{q}$ in SCHA. The solution exists in the range $0<\eta=\frac{\left\langle V^{\prime \prime}\right\rangle}{M \omega_{q}^{2}} \leq$ 0.3. Notice that this domain excludes the regular harmonic phonons (i.e., $\left\langle V^{\prime \prime}\right\rangle=M \omega_{D}^{2}$ ) as it should.

This range for $\eta$ corresponds to $0<\kappa_{q} / \omega_{q} \leq 0.33$ which is also within the range of validity of Eq. (8). In Figs. 1 and 2 this range also corresponds to the monotonically increasing region of $\Delta$ and $T_{c}$ with $\kappa$. The simple argument above shows that squeezed correlated phonon ground state may possibly exist even in certain conventional superconductors. To get a qualitative feeling, we examined the possibility of the self-consistent solution of (11) in a real example. The dimensionless constant $\eta$ and $\kappa / \omega_{D}$ are calculated for lead as a strong-coupling superconductor. Using the Born-von Kármán method, Brockhouse et al. ${ }^{31}$ determined the force constants of $\mathrm{Pb}$ from the phonon-dispersion measurement along the $[\xi \xi \xi],[\xi 00]$ directions for the $L$ and $T$ acoustic branches at $T=100$ $\mathrm{K}$ by the neutron spectrometry. The determined values of $\left\langle V^{\prime \prime}\right\rangle$ and $M \omega_{q}^{2}$ at the zone boundaries at each direction as well as the calculated dimensionless constant $\eta$ and $\kappa / \omega_{D}$ are given in Table I. We find a self-consistent dynamical solution with $\kappa / \omega_{D} \sim 0.32$ which is the only nontrivial solution within the domain where a solution exists (i.e., $0<\eta \leq 0.3$ ). Extrapolation of this result to $T=0 \mathrm{~K}$ is not trivial. However qualitatively the temperature dependence of $\left\langle V^{\prime \prime}\right\rangle$ and $\omega_{D}^{2}$ are expected to be quite similar. Near $T \sim 0 \mathrm{~K}$, therefore the situation is most probably similar to Table I.

The ion-mass dependence in Eq. (11) is determined by $\left\langle V^{\prime \prime}\right\rangle / M \omega_{D}$. Depending on the form of the ion potential and the phonon ground state we assume that $\left\langle V^{\prime \prime}\right\rangle \propto M^{\gamma}$, hence, $\kappa \propto M^{\gamma-1 / 2}$. The corresponding solution of the isotope exponent from Eq. (10) is monotonic as a function

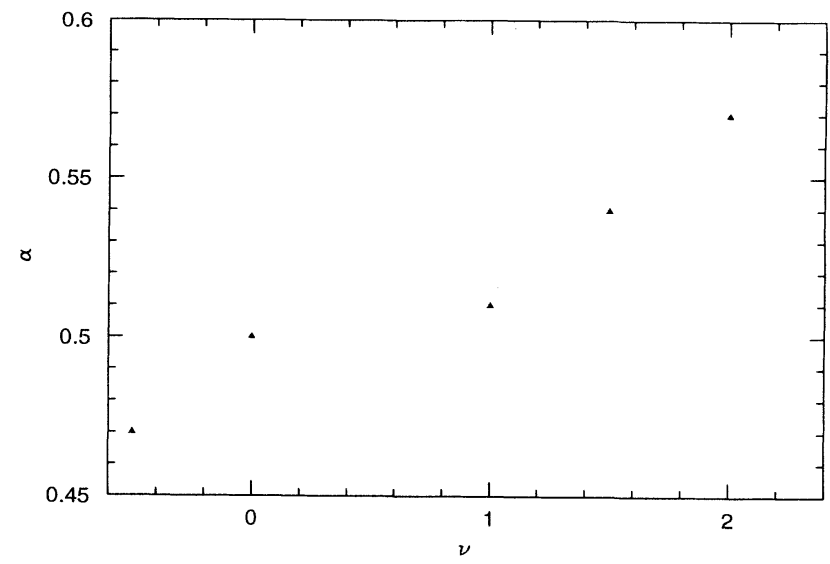

FIG. 4. The isotope exponent $\alpha$ as a function of $\nu=\gamma-1 / 2$.

of $M$ and weakly dependent on $\nu=\gamma-1 / 2$ as shown in Fig. 4.

\section{FINAL REMARKS}

Phonon correlations can be physically realized in a number of systems in different forms. A historical and the simplest example is readily provided by the Peierls transition. There the static change in the $1 \mathrm{D}$ crystal symmetry properties can be visualized as finite number of phonons existing in the zero-temperature phonon ground state. Phonon correlations can be crucial to understand the mechanisms behind the static lattice deformations generated by impurities, defects, finite pressure, and doping. Correlated squeezed phonons are known to yield a better ground state than the two-phonon coherent state in the linear doubly degenerate Jahn-Teller effect. ${ }^{32}$ There are strong evidences that all of the above may be relevant in creating unusual properties of the phonon subsystem particularly in the nonconventional superconductors. ${ }^{33}$

In the theory of strong-coupling superconductivity the isotope exponent $\alpha$ is determined in the EliashbergNambu formalism by the McMillan-Allen-Dynes $T_{c}$ equation. ${ }^{34}$ With harmonic phonons $\alpha$ always yields 0.5 and anharmonicities generally lead to smaller and possibly negative values. The Eliashberg theory with a two-well anharmonic ion potential ${ }^{35-37}$ and three-well one $^{38,39}$ can lead to negative values. Another approach with frozen phonons ${ }^{40}$ yields similar isotope results.

TABLE I. Determined values of $\left\langle V^{\prime \prime}\right\rangle$ from the Born-von Kármán analysis of Ref. 28 and $M \omega_{D}^{2}$ for $\mathrm{Pb}$. The calculated values of $\kappa / \omega_{D}$ from Eq. (11) are also listed.

\begin{tabular}{l|lc|c|l|l}
\hline \hline & & $\left\langle V^{\prime \prime}\right\rangle \times 10^{3}(\mathrm{dyn} / \mathrm{cm})$ & $M \omega_{D}^{2} \times 10^{3}(\mathrm{dyn} / \mathrm{cm})$ & $\nu$ & $\kappa / \omega_{D}$ \\
\hline$\xi \xi \xi$ & $T$ & 5.2 & 13.5 & 0.38 & 0 \\
& $L$ & 29.6 & 217.3 & 0.13 & 0.32 \\
$\xi 00$ & $T$ & 6.5 & 10.7 & 0.6 & 0 \\
& $L$ & 29.4 & 65.0 & 0.45 & 0 \\
\hline \hline
\end{tabular}


It is also necessary to mention the recently observed superconductivity in the intermetallic compounds $L \mathrm{Ni}_{2} \mathrm{~B}_{2} \mathrm{C}$ with $L$ describing a lanthanide element (Y, $\mathrm{Ho}-\mathrm{Lu}){ }^{41}$ Electronic-band properties suggest that these new quaternary compounds are closer to the conventional electron-phonon type (with $T_{c}$ in the range of 15-23 K) than to high- $T_{c}$ superconductors. ${ }^{42}$ The layered structure of this compound can support anharmonic correlated phonon modes orthogonal to the Ni-B layers. It is shown by the present authors that the hopping amplitude of the $\mathrm{Ni} d$ electrons in the planes is modulated by anharmonic lattice deformations of $B$ atoms in the orthogonal direction to the Ni planes. ${ }^{43}$ The first contribution appears in the form of the lowest order squeezed phonon mode with longitudinal polarization perpendicular to the planes which has a band narrowing effect on the $\mathrm{Ni} d$ electrons. The positive baric derivatives of $T_{c}$ and the recently measured unusual boron isotope effect ${ }^{44}$ of these compounds can be qualitatively understood in this model.

In conclusion, the squeezed coupling parameter $\kappa$ effectively depends on the ion mass and the concentration of the carriers. But its explicit form is a rather delicate question. ${ }^{45,46}$ In the case of large coherence length $\xi$ (for conventional superconductors approximately $10^{4}$ lattice spacings) only those quantities which are averaged over the coherence volume are important. Thus $T_{c}$ as well as $\kappa$ depend on the average ion mass. The $\xi$ in the high-temperature superconductors and in $\mathrm{C}_{60}$ related compounds are typically in the range of 10-30 $\AA$ so that the coherence volume comprises only few unit cells. ${ }^{47}$ One then has to investigate separately the dependence of physical quantities on a complicated distribution of different ion masses over the unit cell. Therefore for new superconductors the influence of the local lattice deformations is more important than for conventional ones. On the other side, as the adiabatic approximation is weakened in the existence of strong anharmonic e-ph interaction, the displaced-oscillator state does not properly represent the real phonon ground state. Hence, anharmonic coupling not only leads to a finite rigid displacement but also to the deformation of the phonon wave function. Our research is under progress in the direction of formulating the phonon correlations self-consistently in the strong-coupling anharmonic regime using realistic phonon density of states.

\section{ACKNOWLEDGMENTS}

T.H. appreciates useful conversations with Professor N. M. Plakida, Professor T. Altanhan, Professor A. Erçelebi, and Professor I. Kulik. V.I. acknowledges support by the Ministry of Education, Science and Culture of Japan. $\mathrm{He}$ is also grateful for useful discussions with Professor S. Nakajima, Professor Y. Murayama, and Professor S. Maekawa.
* Present address: Institute for Molecular Science, Okazaki National Research Institutes Center, Myodaiji, Okazaki 444 Japan.

${ }^{1}$ J. Bardeen, R. Schrieffer, and L. Cooper, Phys. Rev. 108, 1175 (1957).

${ }^{2}$ E. Maxwell, Phys. Rev. 78, 477 (1950).

${ }^{3}$ C. A. Reynolds et al., Phys. Rev. 78, 487 (1950).

${ }^{4}$ H. Fröhlich, Proc. R. Soc. London Ser. A 63, 778 (1950).

${ }^{5}$ J. Bardeen, Phys. Rev. 79, 167 (1950).

${ }^{6}$ Proceedings of the International Conference LT-20, edited by R. J. Donally [Physica B (to be published)].

${ }^{7}$ J. P. Carbotte, Rev. Mod. Phys. 62, 1027 (1990); V. L. Ginzburg, Physica C 209, 1 (1993).

${ }^{8}$ R. B. Schwartz et al., in Studies of High Temperature Superconductors, edited by A. Narlikar (Nova Science, New York, 1992), Vol. 9, p. 121.

${ }^{9}$ N. F. Mott, Philos. Mag. Lett. 64, 211 (1991); A. S. Alexandrov and N. F. Mott, Rep. Prog. Phys. 57, 1197 (1994).

$10 \mathrm{~J}$. H. Kim and Z. Tesanovic, in Lattice Effects in High Temperature Superconductivity, edited by Y. Bar-Yam (World Scientific, Singapore, 1992), p. 460.

11 T. Schneider and H. Keller, Phys. Rev. Lett. 69, 3374 (1992).

${ }^{12}$ M. Newns et al., Commun. Condens. Matter Phys. 15, 273 (1992); C. C. Tsuei, et al., Phys. Rev. Lett. 69, 2134 (1992).

${ }^{13}$ N. M. Plakida, in Electron-Phonon Interaction in Oxide Superconductors, edited by R. Baquero (World Scientific, Singapore, 1991).

${ }^{14}$ R. Feile et al., Z. Phys. B 73, 155 (1988); M. Cardona,
Physica C 185-189, 65 (1991); C. Thomsen et al., Solid State Commun. 78, 727 (1991).

${ }^{15}$ C. Foster et al., Physica C 162-164, 1107 (1989).

${ }^{16}$ D. Mikailović et al., Phys. Rev. B 42, 1989 (1990).

${ }^{17}$ V. I. Kudinov et al., Phys. Lett. A 151, 358 (1990).

${ }^{18}$ M. Arai et al., in Phonon Scattering in Condensed Matter VII, edited by M. Meissner and R.O. Pohl (Springer-Verlag, Berlin, 1993), p. 191; Phys. Rev. Lett. 69, 359 (1992).

${ }^{19}$ S. J. Nettel and R. K. Maccrone, Phys. Rev. B 47, 11360 (1993).

${ }^{20}$ Zheng Hang, Phys. Rev. B 38, 11865 (1988).

${ }^{21}$ A. Alexandrov and J. Ranniger, Phys. Rev. B 23, 1796 (1981).

${ }^{22}$ B. K. Chakraverty et al., Solid State Commun. 64, 1147 (1987).

${ }^{23}$ R. Loudon and P. L. Knight, J. Mod. Opt. 34, 709 (1987).

${ }^{24}$ C. F. Lo et al., Phys. Rev. B 50, 418 (1994); C. F. Lo and R. Sollie, Phys. Lett. A 169, 91 (1992).

${ }^{25} \mathrm{~J}$. Ruvalds and A. Zawadowski, Phys. Rev. B 2, 1172 (1970).

${ }^{26}$ L. Pintschovius, Adv. Solid State Phys. 30, 183 (1990).

${ }^{27}$ A self-consistent treatment of phonon correlations in superconductivity is possible using the strong coupling Eliashberg formalism and will be presented elsewhere. In this simple model we consider $\kappa_{q}$ as a given parameter of the phonon subsystem.

${ }^{28}$ E. G. Brovman and Yu. Kagan, Sov. Phys. JETP 25, 365 (1967).

${ }^{29}$ P. Brüesch, Phonons: Theory and Experiments (SpringerVerlag, Berlin, 1982), Vol. I. 
${ }^{30}$ T. Hakioḡlu (unpublished).

${ }^{31}$ B. N. Brockhouse et al., Phys. Rev. 128, 1099 (1962).

${ }^{32}$ Hai Tao Huang and H. Zheng, Solid State Commun. 88, 601 (1993); C. F. Lo, Phys. Rev. A 43, 5127 (1991).

${ }^{33}$ N. M. Plakida, High Temperature Superconductors (Springer-Verlag, Berlin, 1994).

${ }^{34}$ J. P. Frank, in Physical Properties of High Temperature Superconductors, edited by D. M. Ginsberg (World Scientific, Singapore, 1994), Vol. IV, p. 189.

${ }^{35}$ B. N. Ganguly, Z. Phys. 265, 433 (1973).

${ }^{36}$ T. Galbaatar et al., Physica C 176, 436 (1991).

${ }^{37}$ A. P. Zhernov et al., Superconductivity 5, 589 (1992).

${ }^{38}$ Y. H. Crespi and M. L. Cohen, Phys. Rev. B 43, 12921 (1991); 44, 4712 (1991).

${ }^{39}$ Y. H. Crespi and M. L. Cohen, Solid State Commun. 83, 427 (1992); Phys. Rev. B 48, 398 (1993).

${ }^{40}$ K. Nasu, Phys. Rev. B 35, 1748 (1987).
${ }^{41}$ R. J. Cava et al., Nature (London) 367, 252 (1994); T. Siegrist, ibid. 367, 254 (1994); C. Mazumdar et al., Solid State Commun. 87, 413 (1993); R. Nagarajan et al., Phys. Rev. Lett. 72, 274 (1994).

${ }^{42}$ L. F. Mattheiss et al., Solid State Commun. 91, 587 (1994); L. F. Mattheiss, Phys. Rev. B 49, 13279 (1994).

${ }^{43}$ T. Hakioḡlu, V. A. Ivanov, A. S. Shumovsky, and B. Tanatar, Physica C 234, 167 (1994); Proceedings of M2SHTSC-IV, Grenoble, France, 1994 [Physica C 235-240, 2343 (1994)].

${ }^{44}$ D. D. Lawrie and J. P. Franck, Proceedings of M2S-HTSCIV, Grenoble, France, 1994 (Ref. 43), p. 1503.

${ }^{45}$ C. Yu and P. W. Anderson, Phys. Rev. B 29, 6165 (1984).

${ }^{46}$ A. P. Zhernov and A. E. Trenin, Sov. Phys. Solid State 30, 783 (1988).

${ }^{47}$ V. A. Ivanov and Y. Murayama, Physica C (to be published). 